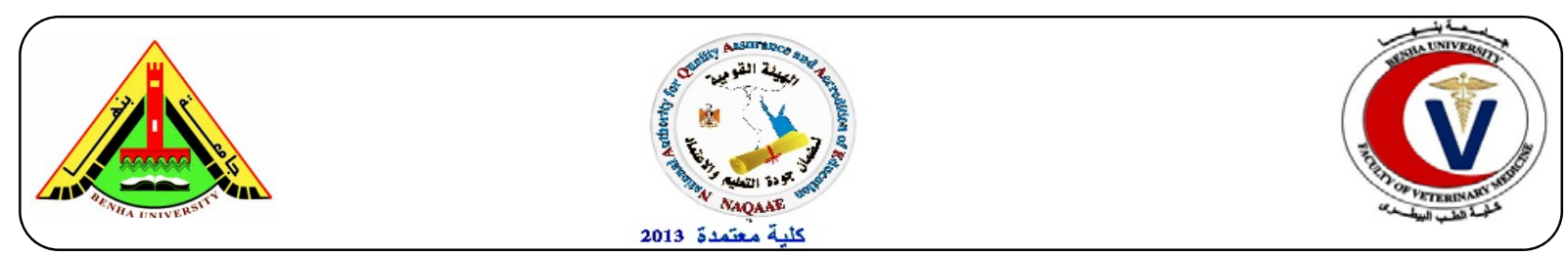

\title{
Effect of natural nanoparticles products on hepato-renal functions in breast carcinoma female rats
}

\author{
Omayma A.R. Abou Zaid ${ }^{1}$, Fatma A. Ahmed ${ }^{2}$, Abdel Fatah M. Badwi ${ }^{3}$, Naglaa M.H. Ibrahim ${ }^{2}$ \\ ${ }^{1}$ Clinical Biochemistry Department - Faculty of Veterinary Medicine-Benha University. \\ ${ }^{2}$ Phyto-chemistry Department-Medical and Aromatic Planets Department- Desert Research Center. \\ ${ }^{3}$ Applied Chemistry Department - Egyptian Petroleum Research Institute
}

\begin{abstract}
A B S T R A C T
The aim of this study was to evaluate the effect of natural nano-composite products in combination with doxorubicin chemotherapy treatment on serum biochemical profile in mammary cancer induced in fifty female rats divided as following; control normal $\mathrm{n}=10$, mammary carcinogenesis Induced group with MNU (50 mg/ kg. b. wt. / I.P dose) n=40 which divided into three subgroups (10 rats in each group): mammary carcinoma non-treated gp, doxorubicin treated group (33 mg / kg. b .wt. / I.P. dose), basic nano-curcumin ( $35 \mathrm{mg} / \mathrm{kg}$. b. wt. /oral dose) + doxorubicin treated group and basic nano-brown algae $(70 \mathrm{mg} / \mathrm{kg}$. b .wt. / oral dose $)+$ doxorubicin treated group. Blood urea nitrogen (BUN) and serum creatinine level, AST and ALT activities were determined during the course of chemotherapy treatment among different groups. The level of BUN, creatinine and AST activity were more than normal reference range in doxorubicin chemotherapy treatment, basic nano-curcumin and basic nano-brown algae; while the value of serum ALT was reported to be within the normal reference range in case of treatment by using basic nano-curcumin with doxorubicin chemotherapy. In conclusion, the obtained results suggest that, treatment with chemotherapy and basic nano-curcumin in combination improve serum biochemical profile in breast carcinoma induced in rats.
\end{abstract}

Keywords: Breast cancer, Biochemical profile, chemotherapy, BUN, Creatinine, AST, ALT.

(http://www.bvmj.bu.edu.eg)

(BVMJ-33(2): 99-106, DECEMBER, 2017)

\section{INTRODUCTION}

Breast carcinoma is the most common malignancy which cause of death in women worldwide (Globocan, 2008). Breast cancer is differ in its clinical, genetic and biochemical profile. Cancer treatments like chemotherapy and radiation therapy generally destroy the cancerous cells in the body. However, some of the normal cells are also sensitive to these treatments and get also damage. Blood chemistry panel (BCP) is a common tests used to estimate a diversity of chemical components released from body tissues or produced during the breakdown or metabolism of certain substances. The blood chemistry panel (BCP) measures the levels of chemicals, enzymes, and organic waste products found in the blood. It determines the healthiness and proper functioning of various organs during chemotherapy treatment. The abnormal blood chemistry results indicate the delay in health status. (Wyld et al., 2003 \& Brown et al., 2012). Chemoprevention is a promising anticancer approach with reduced secondary effects in comparison to classical chemotherapy. Most chemo preventive agents known until today are plant extracts. Curcumin extracted from (Curcuma longa L.), research over the last few decades has shown that curcumin is a potent anti-inflammatory agent with strong therapeutic potential against a variety of cancers (Aggarwal et al., 2003). Curcumin has been found to have a hepatoprotective characteristic similar to silymarin. Animal studies have demonstrated its hepatoprotective effects from a variety of hepatotoxic agents, including carbon tetrachloride $\left(\mathrm{CCl}_{4}\right)$, acetaminophen (paracetamol), and Aspergillus aflatoxin (Park, 2000). In the 1940s, natural product research interest was directed towards marine flora as potential sources in the production of metabolic constituents possessing antimicrobial, antiviral and antifungal properties, besides their effective pharmaceutical and economic properties (Ali and Pervez, 2003). The effect of sulfated polysaccharides from Sargassum spp. repairs the activities of hepatic marker enzymes as it decreases the levels of lipid peroxidation. Also, sulfated polysaccharides administration minimized the oxidants production 
by scavenging the free radicals (Josephine et al., 2008). Recently, targeted and triggered drug delivery systems accompanied by nanoparticle technology have emerged as prominent solutions to the bioavailability of therapeutic agents especially substances which in their natural form have no solubility in the water (as curcumin and brown algae). Accordingly, the effect of natural nanoparticles products on hepato-renal functions in mammary cancer induced in rats was investigated.

\section{MATERIALS \& METHODS}

\subsection{Extraction of Curcumin from turmeric plant rhizomes}

The fresh rhizomes were procured from Sekem Company Farms (Belbeis-Sharqya), Egypt, dried and crushed. The powder was stored in a clean closed container until further use. The dried powder of turmeric $(500 \mathrm{~g})$ was placed in the collar of Soxhlet apparatus $+(1000 \mathrm{ml})$ of methanol as a solvent. The extraction was continued till clear solvent was seen in the collar. The extract was transfer to rotary evaporator to evaporate the solvent residue. The percentage yield of the extract was calculated using the following equation: Yield $(\%)=$ Initial wt. of the dried powder of turmeric / Final wt. of the dried extract $\times 100$ The extracts were kept in the refrigerator till further use.

\subsection{Brown Algae (Sargassum Subrepandum)}

The dried and crushed samples of brown algae from the coast of the Ras Sedr, Red Sea, South of Saini, Egypt. The powder was stored in a clean closed container until further use.

\subsection{Chemicals}

Sodium Bicarbonate Purchased from ElGomhoria Chemicals Company. (MNU) NNitroso-N-Methyl Urea (Chemical Carcinogen): Also known as Nitroso-methyl-urea or N-methyl N-nitrosourea. MNU (purity 99\%) was factorymade by Sigma Chemical Co. (St. Louis, Mo, USA) and purchased from Schnelldorf, Germany through the Egyptian International Center for Import Cairo, Egypt.

\subsection{Doxorubicin (Chemotherapy):}

Synonyms: Adricin, each vial $(25 \mathrm{ml})$ was contained Doxorubicin $\mathrm{HCl}(50 \mathrm{mg})$ manufactured by EIMC United Pharmaceuticals, Badr City, Cairo, Egypt and purchased from EL-Ezaby Pharmacy, Cairo, Egypt.
2.5 Preparations and Measurements of novel nano-composites (basic nano-curcumin \& basic nano-brown Algae):

To prepare proper nanoparticles, $1 \mathrm{M}$ (curcumin, brown algae separately) with low solubility in water was mixed with $4 \mathrm{M}$ sodium bicarbonate buffer, then grinded using mechanical ball mill (350 round/Sec) for $8 \mathrm{hrs}$. The nanoparticles were then dispersed into $50 \mathrm{ml}$ of distilled water making aqueous solution, which was filled in a reactor that was immersed in a water bath adjusted at $11^{\circ} \mathrm{C}$. Afterwards, this reactor was placed in an ultrasound apparatus (VCX-750 commercial sonicator) and sonication was applied in continuous mode at 100 Watt in a glass reaction vessel with thin and indented bottom for uniform and more efficient energy transmission. TEM image was taken (Transmission electron microscope). The TEM studies was represented the TEM image of the novel nano-composites ranging from $6.87-12.7 \mathrm{~nm}$ in diameter and has spherical shape.

\subsection{Experimental Animals:}

Fifty Virgin Female Sprague Dawley rats , 4 weeks old, weighing 100-120 g were purchased from "The Laboratory Animals Research Center", Faculty of Veterinary Medicine, Benha University, and housed in separate wire mesh cages, exposed to good ventilation, humidity and to a 12-hr light/dark cycle .Constant supplies of standard pellet diet, fresh and clean drinking water were supplied ad-libitum. Rats were kept at constant environmental and nutritional conditions throughout the period of the experiment. The animals were left for 15 days for acclimatization prior to the beginning of the experiment.

\subsection{Induction of mammary tumor}

Female rats were injected intraperitoneally with $(\mathrm{MNU})$ at a dose rate of $(50 \mathrm{mg} / \mathrm{kg}$ body weight $)$ five times periodically at 50,70, 80, 90 and 110 days of age (Saminathan et al., 2014).

\subsection{Administration of Doxorubicin (chemotherapy)}

Doxorubicin was administrated at a dose $(33 \mathrm{mg}$ /kg.b.wt. / i.p) dissolved in physiological saline once a week for 4 week (Ihab and Ahmed, 2006).

\subsection{Experimental Design:}

Rats were randomly divided into two main groups, placed in individual cages and classified as follow:

Group (1): Control normal group: comprised 10 rats, received physiological saline contain $1 \%$ 
tween 80 orally, provided with a constant supply of standard pellet diet and plenty of fresh, clean drinking water ad-libitum. Group (2): Induced mammary carcinogenesis group: comprised 40 rats, administered with MNU (I.P) for induction of mammary cancer at a dose level of $(50 \mathrm{mg} / \mathrm{kg}$. b. wt.). This group was divided into three subgroups: Sub-gp-1 (mammary cancer non-treated group) $(\mathrm{n}=10)$ : Considered as mammary carcinogenesis for non-treated group to comparison with two nano-composites treated groups. Sub-gp-2 (doxorubicin treated group) ( $\mathrm{n}=10)$ : rats with mammary cancer were treated with chemotherapy only (Doxorubicin dosage $33 \mathrm{mg} / \mathrm{kg}$. b .wt. /I.P.). Sub-gp-3 (basic nano-curcumin + doxorubicin treated group) ( $\mathrm{n}=10)$ : Rats were administrated with Basic Nano-curcumin orally $(35 \mathrm{mg} / \mathrm{kg}$ by wt. / day) dissolved in water one time per day + doxorubicin $(33 \mathrm{mg} / \mathrm{kg}$. b .wt. /I.P./ one time a week/ 28 days) until the end of the experiment. Sub-gp-4 (basic nano-brown algae + doxorubicin treated group) $(\mathrm{n}=10)$ : Rats were administrated with Basic Nano-brown algae orally and daily (70 $\mathrm{mg} / \mathrm{kg}$. b. wt.) dissolved in water one time per day + doxorubicin $(33 \mathrm{mg} / \mathrm{kg}$. b .wt. /I.P./ one time a week/ 28 days) until the end of the experiment.

\subsection{Blood Samples:}

Blood samples were collected from all animal groups from the retro- orbital venous plexus located at the medial canthus of the eye by using heparinized capillary tubes and collected in dry, clean test tubes. Clear sera were separated by centrifugation at 3500 r.p.m. for $15 \mathrm{~min}$. and then received in Eppendorf's tubes using automatic micropipettes. All serum samples were analyzed for the following parameters BUN, creatinine, ALT and AST.

\subsection{Biochemical parameters}

BUN, serum creatinine, ALT and AST activities were determined according to the methods (Young, 1990 and Murray Charles, 1984) respectively.

\subsection{Statistical analysis}

It was carried out using ANOVA test with two factors under significance level of 0.05 for the whole results using CoSTAT.

\section{RESULTS}

Table 1: represents the Blood Urea Nitrogen (BUN) \& Creatinie values ( $\mathrm{mg} / \mathrm{dL})$, which the level of blood urea nitrogen (BUN) was observed to be more than normal range $(7-20 \mathrm{mg} / \mathrm{dl})$. The value of blood urea nitrogen before the start of chemotherapy (TBR gp) courses was found to be $40 \mathrm{mg} / \mathrm{dL}$. No significant difference of blood urea nitrogen level was noted through chemotherapy treated group and basic nano-curcumin + chemotherapy treated group, while there was significant value noted between the previous groups and basic nano-brown algae + chemotherapy treated group which indicate increase in BUN value. The mean value of creatinine during the chemotherapy treatment of breast cancer group observed to be increased than normal reference range $(0.6-0.8 \mathrm{mg} / \mathrm{dl})$. The value of creatinine was noted to be $1.44 \mathrm{mg} / \mathrm{dL}$ before the start of chemotherapy (TBR gp). No specific alterations were observed in creatinine level between the chemotherapy treated group and basic nano-curcumin + chemotherapy treated group. Also no significant values were noted between the chemotherapy treated group and basic nano-brown algae + chemotherapy treated group.

Table 2: shows the AST \& ALT (U/L) values, which the value (AST or SGOT) level before the start of chemotherapy treatment (TBR) was noted to be $211.66 \mathrm{U} / \mathrm{L}$ above normal reference range ( 0 - 80U/L). Also, AST level was increased among the chemotherapy treated groups further than normal range, but no significant difference found between the chemotherapy treated group and basic nanocurcumin + chemotherapy treated group. The mean value of alanine aminotransferase (ALT or SGPT) level was reported to be within normal reference range ( 0 - $40 \mathrm{U} / \mathrm{L})$ among (NTBR). The value was noted to be $126.33 \mathrm{U} / \mathrm{L}$ before the start of treatment (TBR). Also, an increasing level of ALT enzyme was reported among the chemotherapy treated group. However, a decreasing level of ALT was reported among basic nano-curcumin + chemotherapy treated group, which showed significant difference between the chemotherapy treated group and basic nano-brown algae + chemotherapy treated group.

\section{DISCUSSIONS}

The analysis of blood chemistry provides important information about the function of the kidneys, liver and other organs. Blood urea nitrogen (BUN) is a sensitive indicator of renal abnormalities. The level of creatinine in serum is considered more sensitive kidney function test than BUN by way of kidney injury is the only cause of elevated creatinine as mentioned by (Chauhan, et al., 2016). 
Table 1: Effect of doxorubicin (Dox) alone or in combination with basic nano-curcumin (BNCur+ Dox) or basic nano-brown algae (BNBA+Dox) on BUN and serum creatinine in mammary cancer induced in rats.

\begin{tabular}{ccc}
\hline Groups & BUN mg/dL & Creatinine mg/dL \\
\hline CN & $17^{\mathrm{d}} \pm 1.52$ & $0.33^{\mathrm{d}} \pm 0.17$ \\
MCI & $40^{\mathrm{a}} \pm 2.88$ & $1.44^{\mathrm{a}} \pm 0.01$ \\
MCI+ Dox & $27.66^{\mathrm{c}} \pm 1.45$ & $1.14^{\mathrm{ab}} \pm 0.02$ \\
BNCur+ Dox & $26^{\mathrm{c}} \pm 0.57$ & $1^{\mathrm{bc}} \pm 0.11$ \\
BNBA+Dox & $32.33^{\mathrm{b}} \pm 1.45$ & $1.43^{\mathrm{a}} \pm 0.17$
\end{tabular}

$\mathrm{CN}$ : Control Normal group. MCI: mammary cancer induced in rats group. MCI+ Dox: mammary cancer induced in rats group treated with doxorubicin. BNCur+ Dox: mammary cancer induced in rats group treated with basic nano-curcumin and doxorubicin. BNBA+ Dox: mammary cancer induced in rats group treated with basic nano-brown algae and doxorubicin. Mean values with different super script letters in the same rows are significantly different at $(p<0.05)$.

Table 2: Effect of doxorubicin (Dox) alone or in combination with basic nano-curcumin (BNCur+ Dox) or basic nano-brown algae (BNBA+Dox) on serum ALT and AST enzymes in mammary cancer induced in rats.

\begin{tabular}{ccc}
\hline Groups & ALT U/L & AST U/L \\
\hline CN & $39.66^{\mathrm{d}} \pm 2.72$ & $85^{\mathrm{d}} \pm 2.88$ \\
MCI & $187^{\mathrm{a}} \pm 5.85$ & $211.66^{\mathrm{a}} \pm 15.88$ \\
MCI+ Dox & $126^{\mathrm{b}} \pm 4.25$ & $131.66^{\mathrm{bc}} \pm 14.97$ \\
BNCur+ Dox & $84.33^{\mathrm{c}} \pm 0.41$ & $108^{\mathrm{cd}} \pm 4.48$ \\
BNBA+Dox & $120.66^{\mathrm{b}} \pm 1.54$ & $146^{\mathrm{b}} \pm 14.43$ \\
\hline
\end{tabular}

Figure 1: Effect of different treatments on BUN in mammary cancer induced in rats.

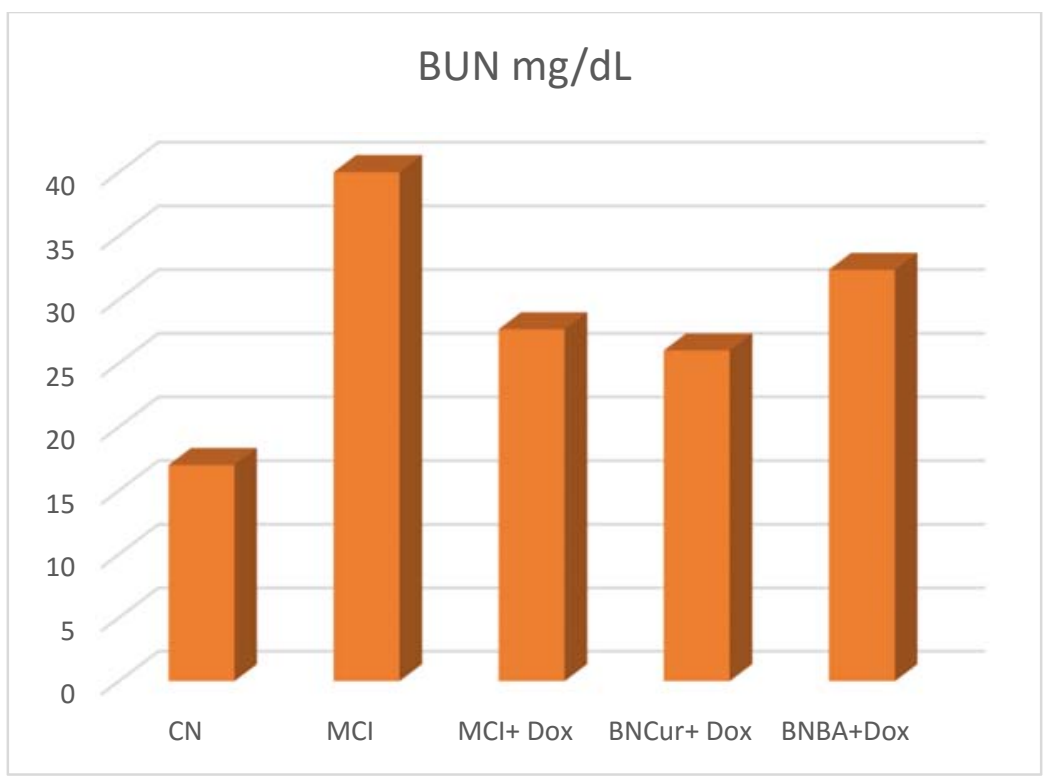

$\mathrm{CN}$ : Control Normal group. MCI: mammary cancer induced in rats group. $\mathrm{MCI}+$ Dox: mammary cancer induced in rats group treated with doxorubicin. BNCur+ Dox: mammary cancer induced in rats group treated with basic nano-curcumin and doxorubicin. BNBA+ Dox: mammary cancer induced in rats group treated with basic nano-brown algae and doxorubicin. 
Figure 2: Effect of different treatments on serum creatinine in mammary cancer induced in rats.

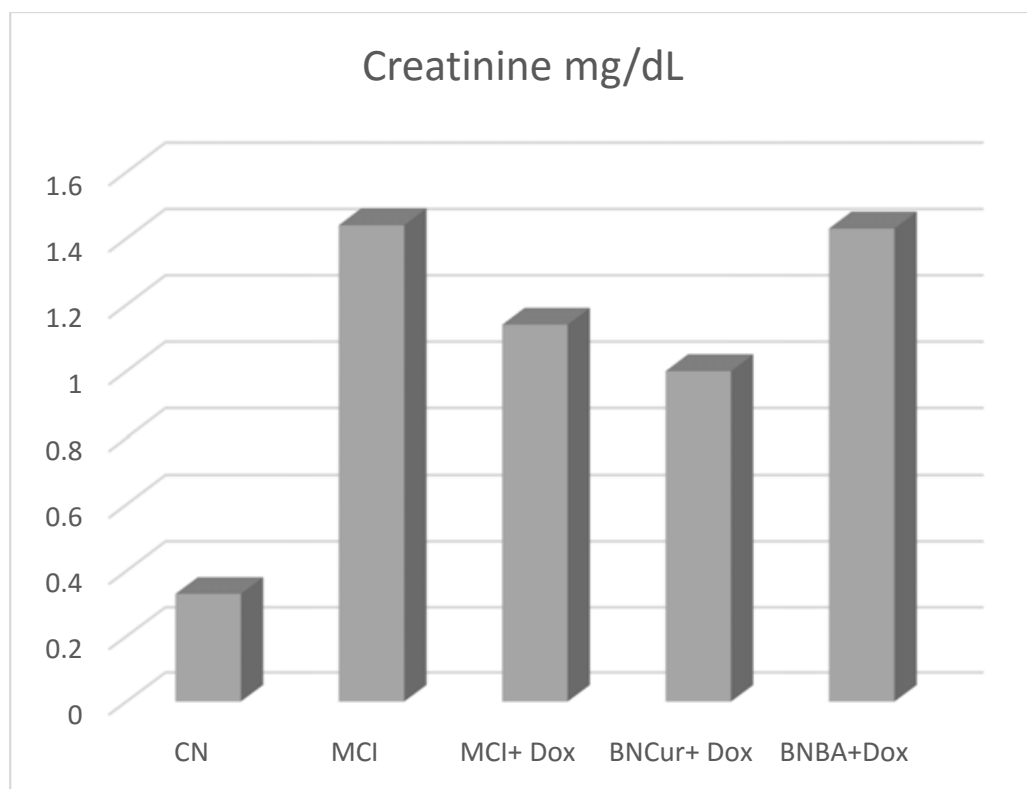

$\mathrm{CN}$ : Control Normal group. MCI: mammary cancer induced in rats group. MCI+ Dox: mammary cancer induced in rats group treated with doxorubicin. BNCur+ Dox: mammary cancer induced in rats group treated with basic nano-curcumin and doxorubicin. BNBA+ Dox: mammary cancer induced in rats group treated with basic nano-brown algae and doxorubicin.

Figure 3: Effect of different treatments on serum AST enzyme (U/L) in mammary cancer induced in rats.

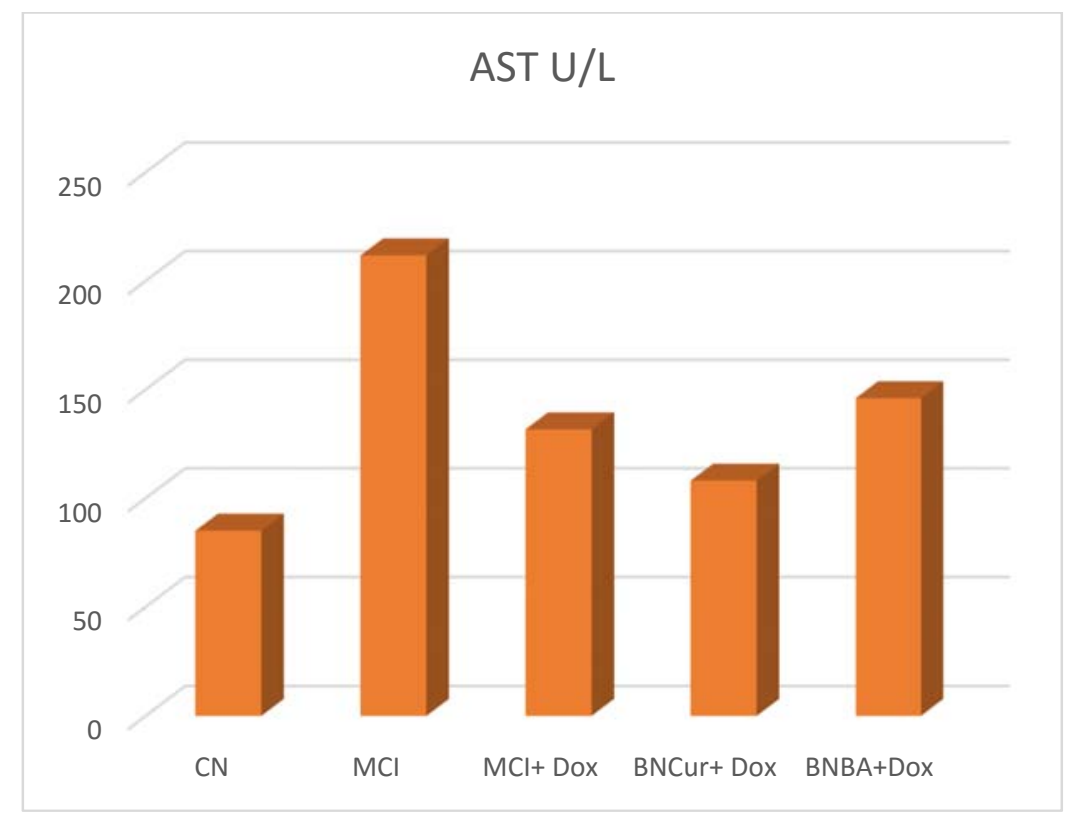

$\mathrm{CN}$ : Control Normal group. MCI: mammary cancer induced in rats group. MCI+ Dox: mammary cancer induced in rats group treated with doxorubicin. BNCur+ Dox: mammary cancer induced in rats group treated with basic nano-curcumin and doxorubicin. BNBA+ Dox: mammary cancer induced in rats group treated with basic nano-brown algae and doxorubicin. 
Figure 4: Effect of different treatments on serum ALT enzyme (U/L) in mammary cancer induced in rats.

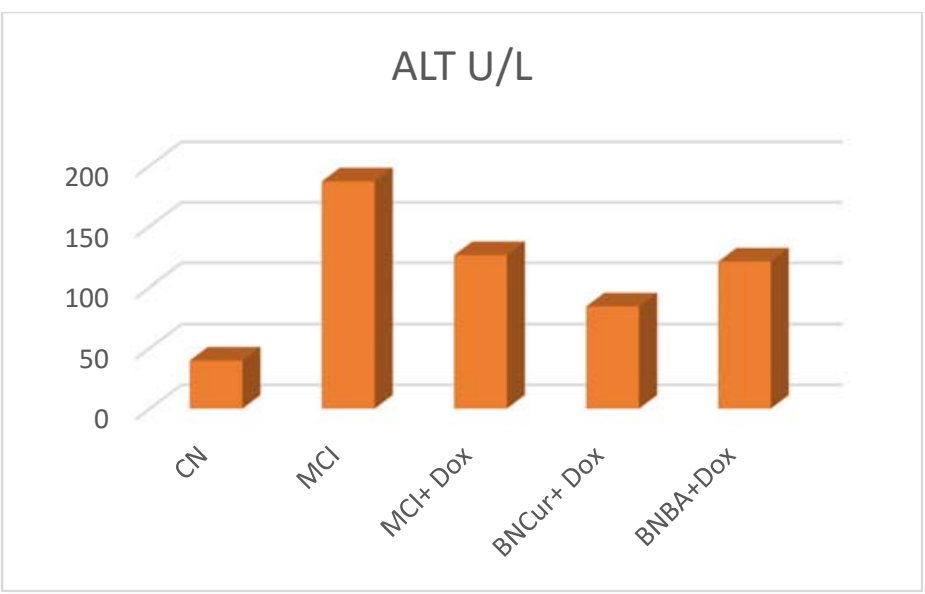

$\mathrm{CN}$ : Control Normal group. MCI: mammary cancer induced in rats group. MCI+ Dox: mammary cancer induced in rats group treated with doxorubicin. BNCur+ Dox: mammary cancer induced in rats group treated with basic nano-curcumin and doxorubicin. BNBA+ Dox: mammary cancer induced in rats group treated with basic nano-brown algae and doxorubicin.

Liver dysfunction related with an overload of hepatotoxicity agents (Navarr and Senior 2006). Hepatotoxicity enhances necrosis, fibrosis, cholestasis, and vascular injury. Liver functioning test (LFT) is mainly based on enzymatic activity of AST and ALT values. Some studies have established that malignant breast cancer patients have increased activities of these transaminases than in benign breast cancer patients (Thangaraju, et al., 1998).

The elevation in serum AST and ALT suggests impairment in liver function which could be due to tumor invasion (George and Zobair, 2010). In the present study, fifty breast carcinoma virgin female Sprague Dawley rats were studied for analytical significance of blood. Biochemical tests were also achieved for chemotherapy treatment group.

During chemotherapy courses the level of blood urea nitrogen (BUN) was observed to be more than normal range $(7-20 \mathrm{mg} / \mathrm{dl})$. Also the mean value of serum creatinine during the chemotherapy treatment of breast cancer group observed to be increased than normal reference range (0.6-0.8 $\mathrm{mg} / \mathrm{dl})$. This results was absolutely agreed with (Chauhan, et al., 2016) which found that, BUN and creatinine levels were reported to be more than normal reference range in contrast to other studies. The cause of the temporarily increasing associated with the presence of chemotherapy and linked to the disappearance of the cause.

Also the value of blood urea nitrogen and serum creatinine values before the start of chemotherapy courses in case of (MCNT gp) was found to be (40 $\mathrm{mg} / \mathrm{dL}, 1.44 \mathrm{mg} / \mathrm{dL}$ ) respectively as shown in
(Table 1), this result was partially consistent with (Ayub et al., 2015) in case of creatinine and varies with him in case of BUN values, which estimated that, the level of serum BUN observed in his investigation was within normal ranges however the level of creatinine was higher than the normal value.

No significant difference pattern of blood urea nitrogen level was noted through doxorubicin treated group and basic nano-curcumin + doxorubicin treated group as shown in, while a specific alterations were observed in creatinine level between the doxorubicin treated group and basic nano-curcumin + doxorubicin treated group as in (Figure 1), these results confirmed with (Parveen, et al., 2017) which demonstrated that curcumin pre-treatment was efficacious in reducing CIS-induced kidney injury in DMBA induced mammary carcinoma in female SpragueDawley rats.

There was significant value noted between the previous groups and basic nano brown algae + doxorubicin treated group which indicate increase in BUN value as shown in (Figure 1). Also no significant values were noted between the chemotherapy treated group and basic nano-brown algae + chemotherapy treated group (Figure 2 ).

The level of enzymes (aspartate aminotransferase, alanine aminotransferase) were analyzed for the proper functioning of liver. Liver function test was used to screen liver infection, to monitor disease progression and possible side effects of medication used. 
The value of Aspartate aminotransferase (AST) level before the start of chemotherapy treatment (mammary carcinogenesis induced group) was noted to be $(211.66 \mathrm{U} / \mathrm{L})$, however; the mean value of AST level was observed to be within normal reference range $(0-80 \mathrm{U} / \mathrm{L})$ as shown in (Table 2$)$.

Also the mean value of alanine aminotransferase (ALT) level was reported to be within normal reference range $(0-40 \mathrm{U} / \mathrm{L})$ among (control normal group), but the value was noted to be $(126.33 \mathrm{U} / \mathrm{L})$ before the start of treatment (MCI gp) (Table 2).

The elevation in AST and ALT values in (TBR gp) was similar to (Rafei et al. 1993), which recorded that; a rise in plasma hepatic enzyme activities were observed in tumor bearing rats is the results of changes in the liver indicated by the incidence of tumor.

However, AST level was increased among the doxorubicin treated groups further than normal range (Figure 3). But no significant difference found between the doxorubicin treated group and basic nano-curcumin + doxorubicin treated group, although significant values were found between the chemotherapy treated group and basic nano-brown algae + chemotherapy treated group (figure 3 ).

An increasing level of ALT enzyme was reported among the doxorubicin treated group (Figure 4), which increase ALT level indicate the inappropriate liver functioning. Conversely, a decreasing level of ALT was reported among basic nano-curcumin + doxorubicin treated group. This result may compatible with (Naik et al., 2010), which showed that curcumin treatment reversed the elevated serum marker enzymes (AST, ALT).

On the other hand, no significant values were reported between the chemotherapy treated group and basic nano-brown algae + chemotherapy treated group (figure 4) this result incompatible with (Yeong-In. et al., 2015) which reported that brown algae may improve the efficacy of chemotherapy drugs for ovarian cancer by enhancing cancer cell apoptosis and reduce nephrotoxicity by protecting against normal kidney cell damage. Therefore, from previous statistical results; treatment by combination with doxorubicin basic nano-curcumin improve serum chemical profile in breast carcinoma rats.

Otherwise, basic nano-brown algae may loss its activity due to its combination with sodium bicarbonate which affect on its active compounds as fucoidan, also it is better to use active ingredients extracted from brown algae rather than using it as crude materials.

\section{CONCLUSION}

Chemotherapy treatment may result in increasing levels of biochemical activities of blood parameters and hence affecting organ system. Increased activity of serum AST and ALT, blood urea nitrogen and serum creatinine directly reflect the effect of different treatments on hepato-renal functions. The study of serum biochemical parameters may be a helpful diagnostic tool in the monitoring of disease, metastasis and different treatment strategies of breast cancer. In the current study, treatment by combination with chemotherapy basic nano-curcumin improve serum biochemical profile in mammary cancer induced rats.

\section{REFERENCES}

Aggarwal, B.B., Kumar, A. and Bharti, A.C. (2003). Anticancer potential of curcumin: preclinical and clinical studies. Anticancer Res. 23: 363-398.

Ali, M.S. and Pervez, M.K. (2003). Secodolastanes from the marine brown alga dictyota dichotoma (Huds.) Lamou. Zeitschrift für Naturforschung, 58b: 438442.

Ayub, A.M.; Inaotombi, D.L. and Lalsanglura R. (2015). Serum biochemical profile of breast cancer patients. European Journal of Pharmaceutical and Medical Research, 6: 210-214.

Brown, JE.; Cook, RJ.; Lipton, A. and Coleman, RE. (2012). Serum lactate dehydrogenase is prognostic for survival in patients with bone metastases from breast cancer: a retrospective analysis in bisphosphonatetreated patients. Clin Cancer Res., 18:63486355.

Chauhan, P.; Yadav, R.; Kaushal, V. and Beniwal P. (2016). Evaluation of serum biochemical profile of breast cancer patients. International Journal of Medical Research \& Health Sciences, 7:1-7.

George, A. and Zobair, M.Y. (2010). When and how to evaluate mildly elevated liver enzymes in apparently healthy patients. Cleveland Clinic Journal of Medicine, 77: 3.

Globocan (2008). Cancer Fact Sheet. Breast Cancer Incidence and Mortality Worldwide in 2008.

Ihab, T. and Ahmed A. (2006). Hesperidin alleviates doxorubicin-induced cardiotoxicity in rats. Levels, SOD activity 
and increase in TBARS levels. Journal of the Egyptian Nat. Cancer Inst., Vol. 21, No. 2, June: 175-184.

Josephine, A.; Nithya, K.; Amudha, G.; Veena, CK.; Preetha, SP. and Varalakshmi, P. (2008). Role of sulphated polysaccharides from Sargassum wightii in cyclosporine Ainduced oxidative liver injury in rats. BMC Pharmacol., 8:1-9.

Murray, Charles. (1984). Losing Ground: American Social Policy, 1950-1980. New York: Basic Box.

Naik, S.R.; Thakare, V.N. and Patil, S.R. (2010). Protective effect of curcumin on experimentally induced inflammation, hepatotoxicity and cardiotoxicity in rats: Evidence of its antioxidant property. Exp Toxicol Pathol., 2: 524-528.

Navarro, VJ.; and Senior, JR. (2006). Drug-related hepatotoxicity". N Engl J Med., 354:731739.

Park, EJ.; Jeon, CH.; Ko, G.; Kim, J. and Sohn, DH. (2000). Protective effect of curcumin in rat liver injury induced by carbon tetrachloride. J Pharm Pharmacol., 52: 437 40.

Parveen, K.; Chandana, C.B.; Kunjbihari, S. and Rajeev, K.S. (2017). Curcumin Ameliorates Cisplatin-Induced Nephrotoxicity and Potentiates Its Anticancer Activity in SD Rats: Potential Role of Curcumin in Breast Cancer Chemotherapy. Front Pharmacol., 8: 132.
Rafei, I.; Fawzeya, M.A. and Mohammed, B. (1993). Possible renal dysfunction effect of nigella sativa seeds in rabbits. J. Biochem. Sci. Therapeutic, 9: 19-25.

Saminathan, M.; Rai, R.B.; Dhama, K.; Ranganath, G.J.; Murugesan, V.; Kannan, K.; Pavulraj, S.; Gopalakrishnan, A. and Suresh, C. (2014). Histopathology and immunohistochemical expression of nmethyl-n-nitrosourea (NMU) induced mammary tumors in Sprague-Dawley rats. Asian Journal of Animal and Veterinary Advances; 9: 621-640.

Thangaraju, M.; Rameshbabu, J.; Vasavi, H.; Ilanchezhian, S.; Vinitha, S. and Sachdanandam, P. (1998). The salubrious effect of tamaxifen on serum marker enzymes, glycoproteins, and lysosomal enzymes level in breast cancer women. Molecular and Cellular Biochemistry; 185:85-94.

Wyld, L.; Gutteridge, E.; Pinder, SE.; James, J.J.; Chan, S.Y.; Cheung, K.L.; Robertson, J.F. and Evans, A.J. (2003). Prognostic factors for patients with hepatic metastases from breast cancer. Br J Cancer.; 89(2):284-290.

Yeong-In, Y.; Ji-H, A.; Youn, S.C. and Jung-Hye, C. (2015). Brown algae phlorotannins enhance the tumoricidal effect of cisplatin and ameliorate cisplatin nephrotoxicity. Gynecologic Oncology; 136(2): 355-364.

Young, D.S. (1990): Effects of Drugs on Clinical Laboratory Tests. Third Edition. 1990: 3: 612. 